\title{
How do we train healthcare professionals to integrate genomics into their practice? MSc in genomic medicine, Swansea University, Wales
}

\author{
Authors: Bryony Coupe, ${ }^{A}$ Maurizio Brotto, ${ }^{B}$ Rhiannon Clegg, ${ }^{C}$ Helen Daniels, ${ }^{A}$ Jason Griffiths, ${ }^{D}$ \\ Torsten Hildebrandt, ${ }^{\mathrm{A}}$ Ruth Young, ${ }^{\mathrm{E}}$ Rachel Thomas $^{\mathrm{F}}$ and Claire Morgan ${ }^{\mathrm{A}}$
}

\begin{abstract}
Introduction
Genomic medicine is transforming the way in which we understand health and disease, particularly rare diseases and cancer. Rapid advances in DNA sequencing are having major implications for patients in terms of diagnosis, treatment and prognosis, provoking a shift from a traditional 'one size fits all' approach to strategies tailored to the individual, known as personalised medicine or precision medicine. ${ }^{1}$ The UK is investing heavily in genomic medicine, with NHS England becoming the first health service in the world routinely offering genomic testing, ${ }^{2}$ and all healthcare professionals will soon be expected to have an understanding of genomics and integrate this into their practice. In recognition of these advances, in 2017 the Welsh government introduced the Genomics for Precision Medicine Strategy, aimed at developing genetics and genomics to improve health and healthcare provision in Wales. ${ }^{3}$
\end{abstract}

\section{Methods}

To help fulfil the Genomics for Precision Medicine Strategy, ${ }^{3}$ Swansea University Medical School launched an MSc in genomic medicine in May 2018. It is currently the only university in Wales to provide this opportunity to multidisciplinary healthcare professionals, imparting the skills and knowledge necessary to interpret genomic data and understand its impact on patient care. Based on the indicative curriculum of Health Education England and Genomics England, this 2-year part-time master's course integrates lectures, workshops, tutorials, interactive group learning and guest lectures, while encouraging and facilitating workplace learning. Modules include bioinformatics, genomics of common and rare inherited diseases, ethics, genomics techniques and optional modules such as pharmacogenomics. Module assessments are summative and students must complete a

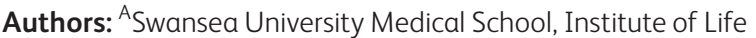
Science; ${ }^{B}$ Abertawe Bro Morgannwg University Health Board; ${ }^{\mathrm{C}}$ Aneurin Bevan University Health Board; ${ }^{\mathrm{D}}$ Hywel Dda University Health Board; ${ }^{E}$ Cardiff and Vale University Health Board; ${ }^{{ }^{B}}$ Betsi Cadwaladr University Health Board, UK dissertation describing an applied genomics project, research or literature review.

\section{Results and discussion}

Eight students completed their first year of study in January 2019. A variety of healthcare professionals enrolled (laboratorybased microbiologists, geneticists, pathologists, general medics, oncologists and paediatricians), which fostered collaborative work and learning. The formal and informal feedback for all modules has been excellent, with students ranking all aspects as 'highly satisfactory'. Students have attended conferences and workshops related to genomic medicine and have disseminated knowledge to their allied healthcare professionals. Their varied dissertation proposals range from integrating new genomics techniques into current NHS practice to facilitating improved patient access to genomics-related healthcare.

\section{Conclusion}

With the future direction of care set out by the NHS and rapidly advancing genomics technology, the need for education and training in the field of genomic medicine is irrefutable. The MSc in genomic medicine at Swansea University School of Medicine plays a vital role in educating healthcare professionals in Wales seeking knowledge and skills in this emerging discipline.

\section{References}

1 NHS England. Improving outcomes through personalised medicine. Leeds: NHS England, 2016. www.england.nhs.uk/wp-content/ uploads/2016/09/improving-outcomes-personalised-medicine.pdf [Accessed 25 March 2019].

2 Hill S. A momentous milestone in the future of the NHS. 20 February 2019. www.england.nhs.uk/blog/a-momentous-milestone-in-thefuture-of-the-nhs/ [Accessed 25 March 2019].

3 Welsh Government. Genomics for precision medicine strategy. Welsh Government, 2017. https://gov.wales/sites/default/ files/consultations/2018-06/summary-document.pdf [Accessed 25 March 2019]. 